Technological University Dublin

DÜBLIN

ARROW@TU Dublin

2009-01-01

\title{
Holographic Recording in Acrylamide Photopolymers: Thickness Limitations
}

\author{
Mohammad Mahmud \\ Technological University Dublin \\ Izabela Naydenova \\ Technological University of Dublin, izabela.naydenova@tudublin.ie \\ Nitesh Pandey \\ Technological University of Dublin
}

See next page for additional authors

Follow this and additional works at: https://arrow.tudublin.ie/cieoart

Part of the Condensed Matter Physics Commons, and the Optics Commons

\section{Recommended Citation}

Mahmud, M. et al. (2009) Holographic recording in acrylamide photopolymers: Thickness limitations. Applied Optics, vol. 48, no.14, pp. 2642-2648. doi:10.1364/A0.48.002642

This Article is brought to you for free and open access by the Centre for Industrial and Engineering Optics at ARROW@TU Dublin. It has been accepted for inclusion in Articles by an authorized administrator of ARROW@TU Dublin. For more information, please contact arrow.admin@tudublin.ie, aisling.coyne@tudublin.ie, gerard.connolly@tudublin.ie.

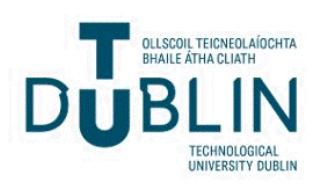




\section{Authors}

Mohammad Mahmud, Izabela Naydenova, Nitesh Pandey, Tzvetanka Babeva, Raghavendra Jallapuram, Suzanne Martin, and Vincent Toal 


\title{
Holographic recording in acrylamide photopolymers - thickness limitations
}

\author{
Mohammad Sultan Mahmud, ${ }^{1, *}$ Izabela Naydenova, ${ }^{1,2}$ Nitesh Pandey, 1 Tzwetanka Babeva, 1 \\ Raghavendra Jallapuram, ${ }^{1}$ Suzanne Martin ${ }^{1}$ and Vincent Toal 1,2 \\ 1) Centre for Industrial and Engineering Optics, Dublin Institute of Technology, Dublin 8, Ireland \\ 2) School of Physics, Faculty of Science, Dublin Institute of Technology, Dublin 8, Ireland \\ Tel: ++353 14027927, *Corresponding author's Email: mohammad.mahmud@dit.ie
}

\begin{abstract}
Holographic recording in thick photopolymer layers is important for application in holographic data storage, volume holographic filters and correlators. Here, the characteristics of acrylamidebased photopolymer layers having thickness from $250 \mu \mathrm{m}$ to 1-mm were studied. For each layer thickness samples with three different values of absorbance were studied. By measuring both the first order diffraction efficiency growth of holographically recorded gratings and studying the diffraction patterns obtained, the influence of scattering on the diffraction efficiency of thick volume holographic gratings was analyzed. It was observed that above a particular thickness and absorbance, the first order diffraction efficiency significantly decreased because of increased holographic scattering. From the first order diffraction efficiency dependence on the samples absorbance and thickness it is possible to choose photopolymer layer properties that are suitable for a particular holographic application. This study was carried out in order to determine the highest layer thickness that could be used in phase code multiplexed holographic data storage system utilizing thick photopolymer layers as a recording medium.
\end{abstract}

Key words: Acrylamide-based photopolymer layer, optical absorption losses, diffraction efficiency, thick photopolymer layers, diffraction pattern, noise grating

\section{Introduction}

Photopolymers [1-10] are considered one of the most versatile holographic recording media due to their high sensitivity, wide dynamic range, and relatively low cost. Many photopolymers have the advantage that they are be self-developing needing no wet processing or thermal treatment. 
However their applications are restricted by the need for thick layers and the material shrinkage that occurs during and after recording [2, 5, 11-13]. In data storage applications this can change the fringe spacing and therefore the reconstruction angle, resulting in no light being diffracted at the expected reconstruction angle and the stored data page cannot be recovered. Very thick photopolymer layers (500 $\mu \mathrm{m}$ or more) are essential for high capacity holographic data storage media [14-18]. Therefore we focus our efforts on developing thick acrylamide-based photopolymer layers characterised by suitable holographic properties [18]. Other research groups studied acrylamide based photopolymer layers (PVA/AA) with different compositions and recorded holograms with a physical thickness of around $1 \mathrm{~mm}[19,20]$. Recording in thick photopolymer layers has two drawbacks; firstly, increased layer thickness leads to increase of the difference between the effective optical thickness and the physical thickness in the material due to the absorption of light [21], and secondly, increased losses due to scattering and absorption.

In this paper we characterize thick layers of acrylamide-based photopolymer developed at the Centre for Industrial and Engineering Optics [5, 6], by measuring holographic parameters such as diffraction efficiency, (DE), angular selectivity and by studying the diffraction patterns obtained. The influence of layer absorbance on the greatest thickness, for which the optical losses in the first diffraction order are acceptable, is discussed.

\section{Theory}

The acrylamide based photopolymer is a self-developing dry layer consisting of monomers, an electron donor or initiator, a photosensitizer and a polymer binder acting as a matrix in which the other components are suspended. Holographic recording in this material is based on photopolymerization reactions in the areas illuminated by laser light of appropriate wavelength. The dye molecules absorb photons and enter into excited states in which they react with the electron donor (initiator) molecules to generate free radicals which then initiate the polymerization process. When a spatially modulated light field is used, polymerization uses up monomer producing a monomer concentration gradient and monomer molecules diffuse from the unexposed regions to the exposed regions where they are polymerized as described. These processes of polymerization and diffusion lead to a spatially modulated change of refractive index of the material and a grating is recorded. Recording may continue until no more monomer is left in the unexposed region or it may be intermittent so that several recordings may be made with 
different spatial frequencies of illuminating light patterns. There are different theoretical models explaining the formation of holographic gratings in photopolymer material [22, 23]. Diffusion studies of acrylamide based photopolymer [24, 25] show that mass transport from dark to bright regions is faster than in other photopolymer systems [26, 27]. Depending upon the thickness of photopolymers layers, noise gratings may be observed due to scattering from inhomogeneities [2, 28-30] in the recording material during exposure. The scattered field can be treated as the superposition of a large number of plane waves each of which interferes with the recording beams, producing weak planar parasitic gratings. When illuminated with a reconstruction beam, the weak gratings diffract some of the incident light. Under exactly the same readout conditions as for recording, all parasitic gratings simultaneously satisfy the Bragg condition and give rise to scatter. Any deviation from the recording conditions, either in wavelength, angular rotation [3133] or polarization state, [34] increases the number of parasitic gratings for which the Bragg condition is violated, decreasing the scattered intensity as a result. As the thickness of the layer increases the amount of optical inhomogeneities also increases and it may be expected that there is some critical thickness beyond which the diffracted beam completely disappears because of the scattering effect. Different compositions of photopolymer layers are expected to have different critical thickness.

\section{Experiment}

\subsection{Preparation of thick layers}

The components of the thick photopolymer layers are acrylamide monomer $(0.6 \mathrm{~g})$ and N,N'methylene-bisacrylamide cross linking monomer $(0.2 \mathrm{~g})$, triethanolamine initiator $(2 \mathrm{ml}), 10 \mathrm{ml}$ polyvinyl alcohol binder (20\% w/v water stock solution) and Erythrosin B sensitising dye $(0.11 \%$ wt. stock solution) $[5,6]$. The amount of dye added to the layer was adjusted to maintain constant absorbance independent of the thickness. The $20 \%$ concentration of PVA was used to enable faster drying of the photopolymer layer. On the other hand, greater PVA concentration increases the viscosity and the time required to make the stock solution. To obtain thick layers the photopolymer solution was deposited in a Petri dish. Once dry the layer was removed and placed on a glass slide for use in recording holographic gratings. To measure thickness using a white light surface profilometer (Micro XAM S/N 8038), the layer was cut back to the glass surface and part of it peeled off. 


\subsection{Experimental setup}

A two-beam holographic optical set-up (Fig.1) was used to record transmission gratings having an interbeam angle of $15^{\circ}$ to record unslanted transmission diffraction gratings using a $\mathrm{Nd}$ $\mathrm{YVO}_{4}$ laser $(532 \mathrm{~nm})$. The gratings were recorded in photopolymer samples of different thicknesses ranging from 250 to $1000 \mu \mathrm{m}$ at recording intensity, $5 \mathrm{~mW} / \mathrm{cm}^{2}$ and spatial frequency of 1000 lines $/ \mathrm{mm}$. The recording intensity was controlled by a variable neutral density filter. The diffracted and transmitted intensities were monitored in real time by probing the grating with a $\mathrm{He}-\mathrm{Ne}$ laser $(633 \mathrm{~nm})$. The photopolymer material is insensitive to this wavelength. Both the probe and recording beams were vertically polarized. The real time diffraction efficiency measurements were made at the Bragg angle.

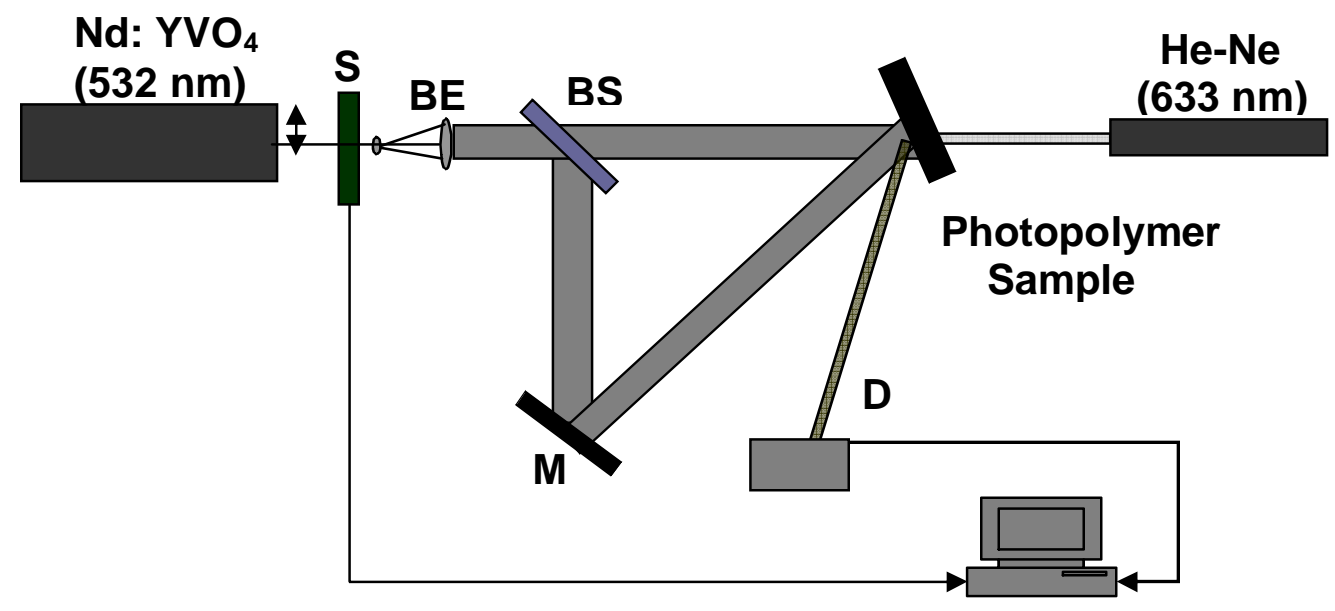

Fig: 1. Experimental setup. Here, S: Solenoid shutter, BE: beam expander, BS: beam splitter, M: mirror, and D: optical power meter.

To measure the diffracted intensity dependence on the incident angle of the probe beam, the grating was placed on a precision rotational stage (Newport, ESP 300, resolution 1000x). The intensity was read by an optical power meter (Newport 1830-C) and the data transferred to a computer. The accuracy of measurement of the Bragg angle was \pm 0.001 degrees. The maximum intensity of the first order diffracted beam was measured and the value obtained was used to correct the diffraction efficiency growth curve for any off-Bragg error arising from shrinkage.

\section{Results and discussion}

\subsection{UV-VIS spectra for same optical absorption (A) but different layer thickness}

The absorption spectra were measured using a UV-VIS-NIR absorption spectrometer (PerkinElmer Lambda 900). Fig. 2 shows the absorption spectra at absorbencies of $0.42,0.17$ and 0.10 
at $532 \mathrm{~nm}$ for thicknesses of $250 \mu \mathrm{m}, 350 \mu \mathrm{m}, 450 \mu \mathrm{m}, 550 \mu \mathrm{m}, 800 \mu \mathrm{m}$ and $1000 \mu \mathrm{m}$. The variation of thickness in each layer is $\pm 10 \mu \mathrm{m}$. The peak absorption is at $535 \mathrm{~nm}$ so a He-Ne laser $(633 \mathrm{~nm})$ was used as a probe beam to avoid influencing the recording process. The same absorbance values at different thicknesses were achieved by using appropriate amounts of the sensitizing dye. It was shown that the absorbance, (A) for layers with thicknesses ranging form 250 to $1000 \mu \mathrm{m}$ varies by only $5 \%$. The slightly higher absorbance of the thicker samples is due to the increased scattering in these samples. Such small variation indicates the generally low level of scattering in the investigated photopolymer.

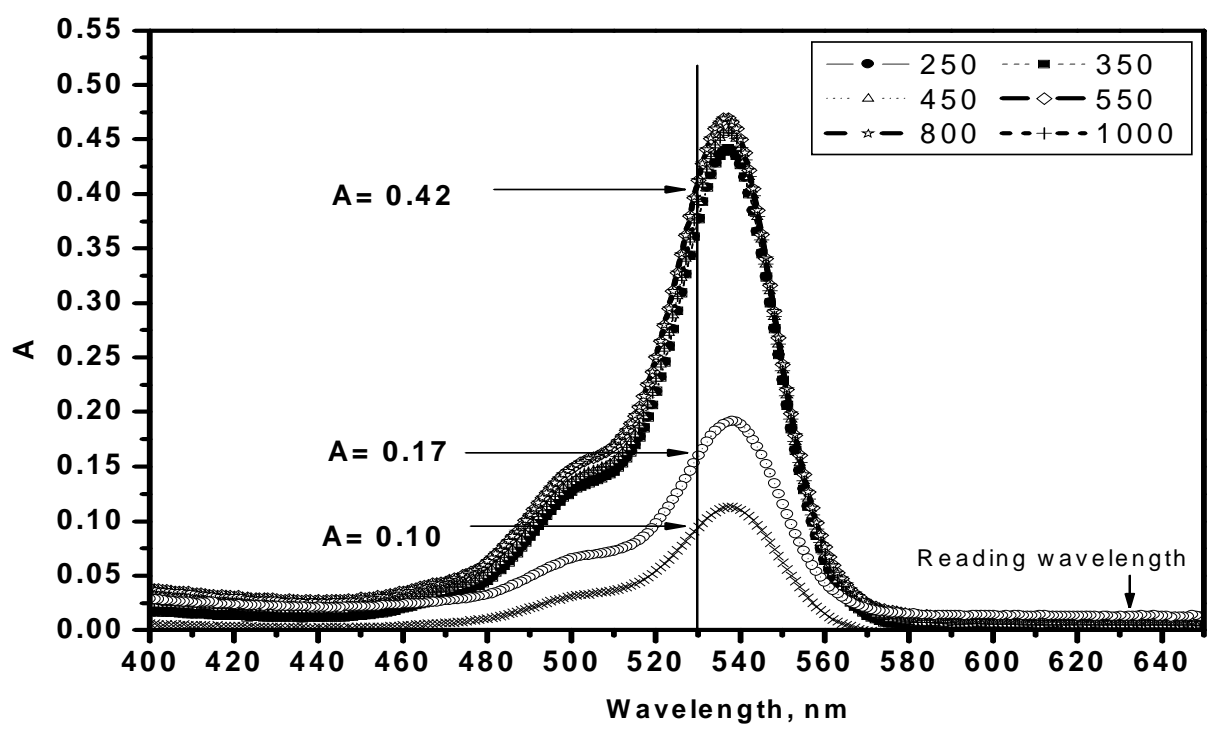

Fig: 2. UV-VIS spectrum measurements for absorbance, $A_{532 \mathrm{~nm}}=0.42$ at different thicknesses of

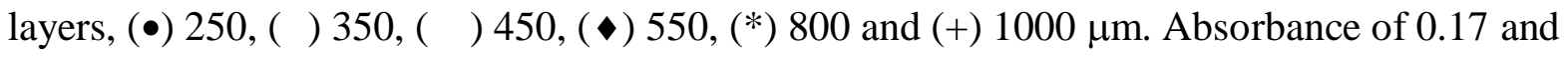
0.10 shown here for comparison.

\subsection{Diffraction Efficiency, (DE) Measurements}

Fig.3 shows the DE versus exposure time for three different absorbencies at different thicknesses $(250 \mu \mathrm{m}, 350 \mu \mathrm{m}, 450 \mu \mathrm{m}, 550 \mu \mathrm{m}, 800 \mu \mathrm{m}$ and $1000 \mu \mathrm{m}$ thick). The DE increases with the exposure and reaches maximum for all thicknesses. As the thickness increases the maximum DE reached gradually starts to decrease mainly due to the noise grating caused by scattering. Further increase of the exposure leads to an oscillatory evolution of DE, typical for over-modulated volume gratings [35] and none of these layers reach $100 \%$ DE due to scattering effect. The final value of diffraction efficiency is reached at exposure times longer than 55 seconds. 

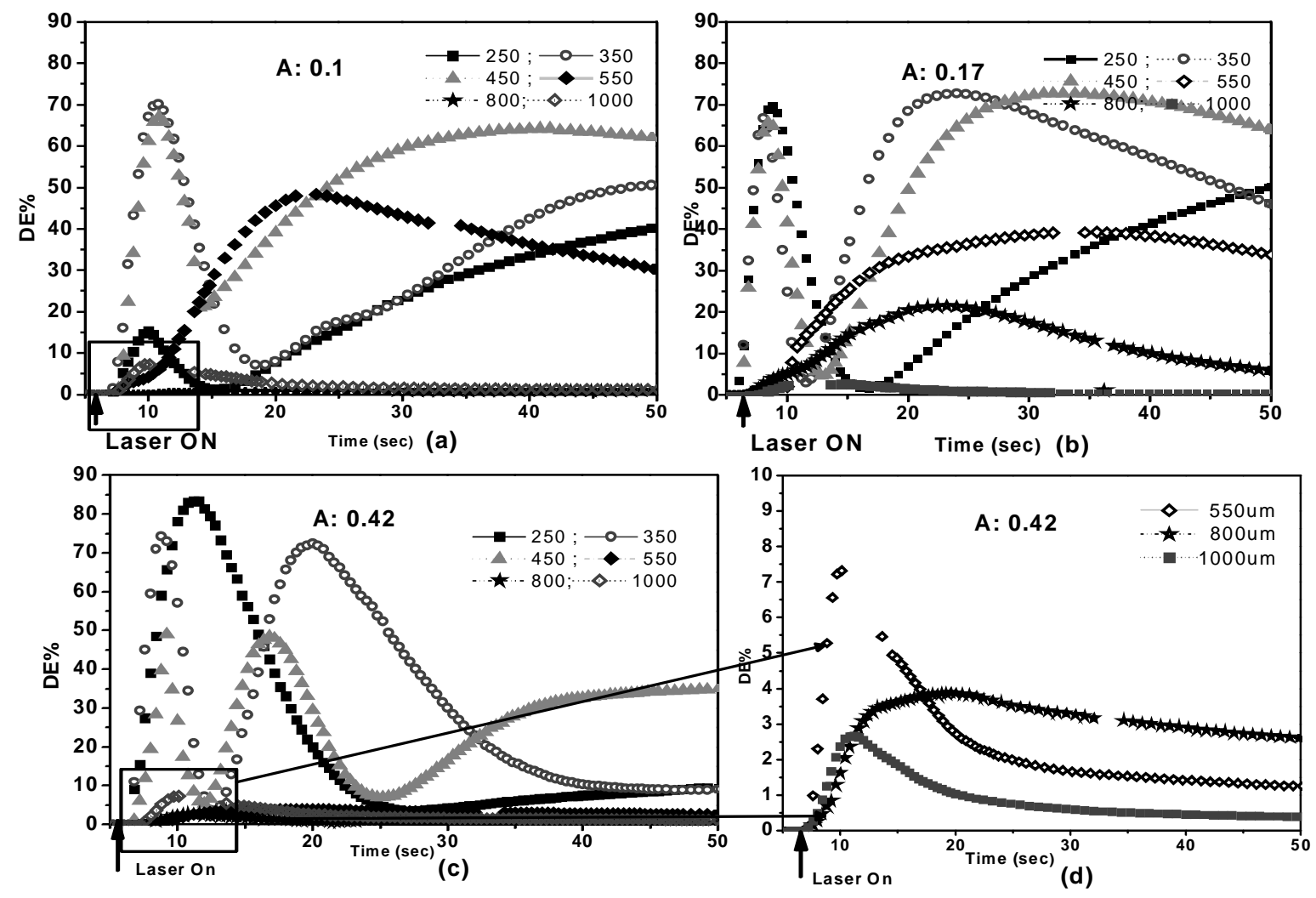

Fig.3: DE vs. exposure time for $\mathrm{A}_{532 \mathrm{~nm}}$ of 0.10 (a), 0.17 (b) and 0.42 (c, d) for different layer

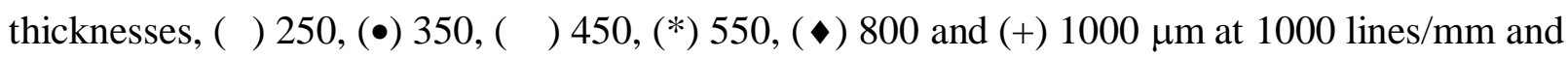
intensity $5 \mathrm{~mW} / \mathrm{cm}^{2}$. Here, (d) is a magnified version of (c).

The results from this experiment can be summarized with the help of Fig.4 in which the dependence of the first maximum of the first order diffraction efficiency on the absorbance of layers with different thickness is presented. It is observed from Fig.4 that in layers of $250 \mu \mathrm{m}$ thickness $\mathrm{A}_{532}>0.17$ is required in order to achieve higher than $80 \%$ diffraction efficiency. Optical losses are relatively low at this thickness.

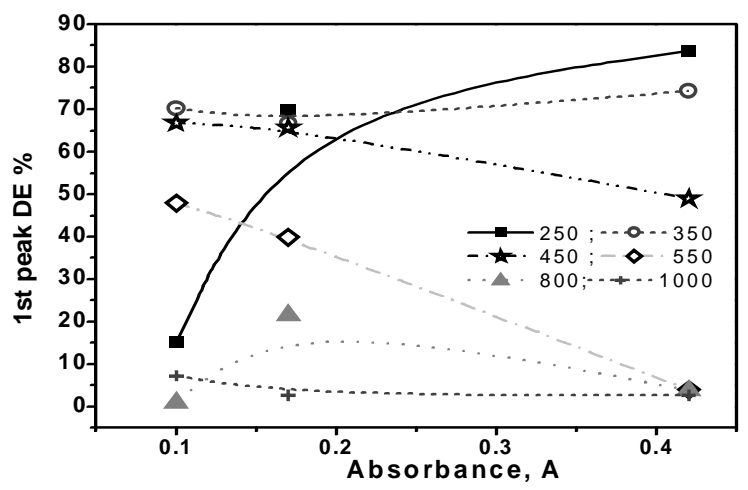

Fig.4: First maximum of the first order DE vs. $\mathrm{A}_{532}$ for different layer thicknesses. 
For layer thickness of $350 \mu \mathrm{m}$, the first maximum of first order diffracted beam is practically independent of the absorbance in the range of 0.1-0.4. This allows for flexible adjustment of the layer sensitivity by varying the dye concentration without significant loss in the first order diffraction efficiency due to holographic scattering. Increase above 0.17 of the absorbance in layers with $450 \mu \mathrm{m}$ thickness leads to substantial deterioration of the diffraction efficiency. At this particular thickness an absorbance lower than 0.17 would not be beneficial and will only lead to a drop in sensitivity of the layers. The first peak of the first order DE decreases almost linearly with increase of the absorbance. Using this dependence one can determine the appropriate layer properties depending on the tolerance to losses in the particular application. For layers of thickness $800 \mu \mathrm{m}$ the optimum absorbance is between 0.17 and 0.4 but the optical losses are very high as seen in Fig.4. layers with thickness $1 \mathrm{~mm}$ performed poorly and further optimization would be required. The main target for optimization could be the PVA matrix. Improvement of the scattering properties could be achieved by choice of the molecular weight and the level of hydrolysis of the PVA.

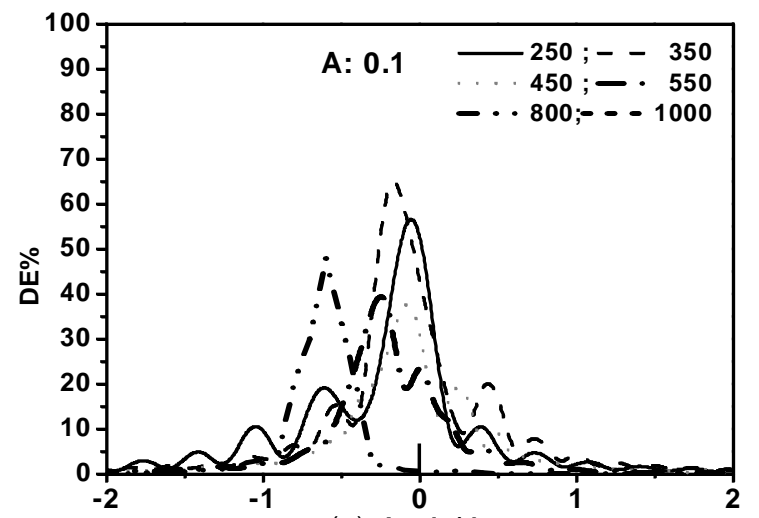

(a) Angle(deg)

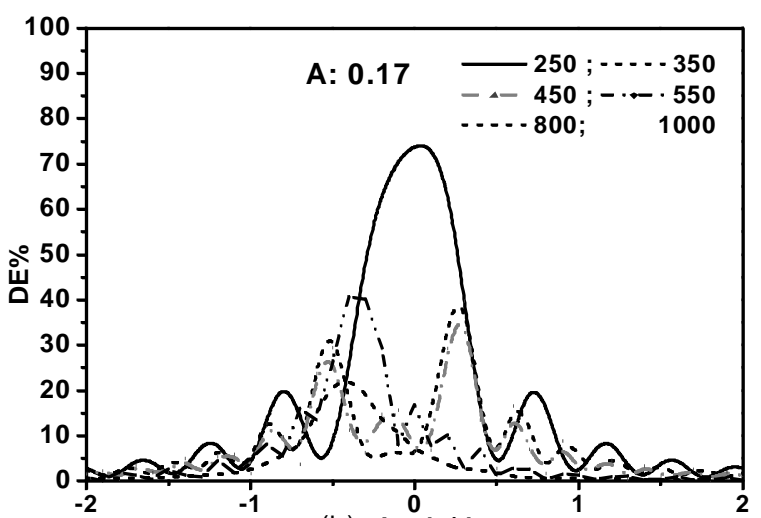

(b) Angle(deg)

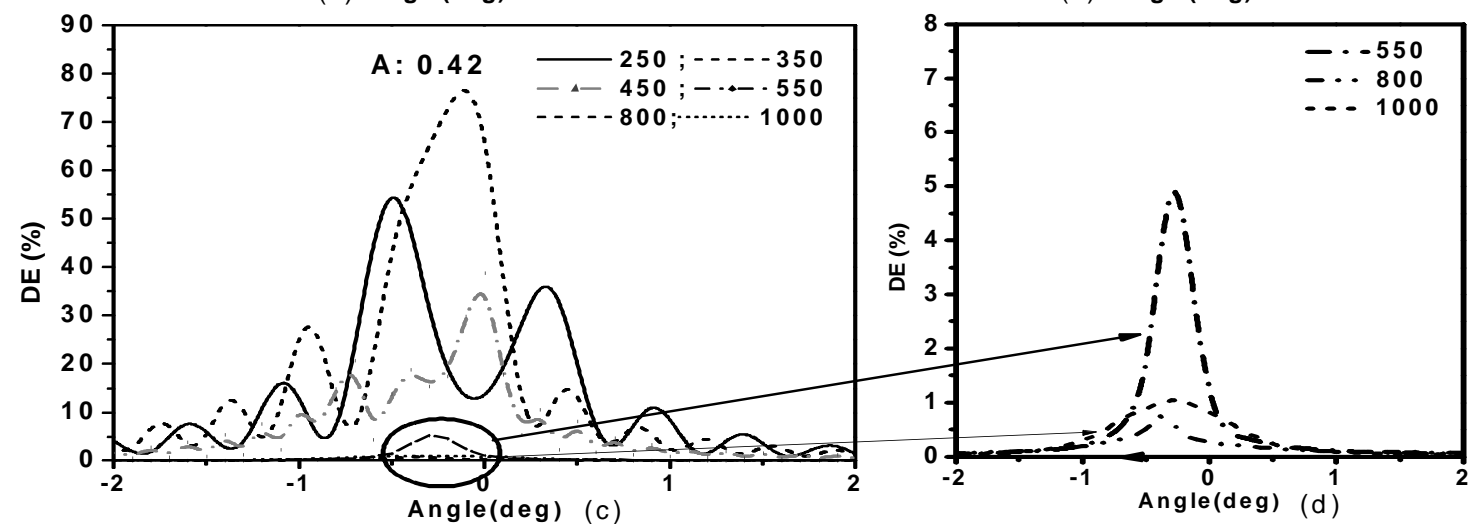

Fig.5. Angular selectivity (Bragg) curves, for $A_{532 \mathrm{~nm}}$ of 0.10 (a), 0.17 (b) and 0.42 (c, d) for

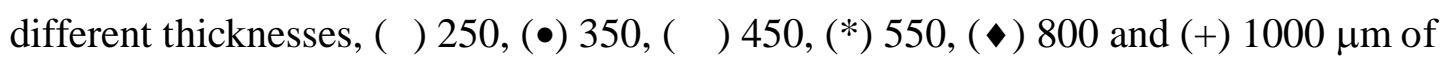
photopolymer layers at exposure intensity of $5 \mathrm{~mW} / \mathrm{cm}^{2}$. 
Fig. 5 shows the angular selectivity curves for the recorded gratings for three sets of absorbances. It is found that maxima of the central and side lobes decrease with increasing layer thickness. The latter completely disappear when the layer thickness is greater than $550 \mu \mathrm{m}$, $800 \mu \mathrm{m}$ and $1000 \mu \mathrm{m}$ for $\mathrm{A}_{532 \mathrm{~nm}}=0.42,0.17$ and 0.10 respectively. Results from other photopolymer compositions suggest a thickness beyond which side lobes vanish due to noise gratings [20, 28-30]. This limits the performance of any thick photopolymer layer. Another possible reason for the attenuation of the side lobes in the Bragg selectivity curves is the nonuniformity of the refractive index profile through the depth of the layer [21]. At the beginning of the recording, the light is attenuated in the material because of the absorption by the sensitizing dye leading to attenuation of the refractive index modulation with depth. As a result the side lobes of the angular selectivity curve disappear [21].

Fig 6 shows the dependence of DE on exposure time for different absorbances and layer thicknesses.
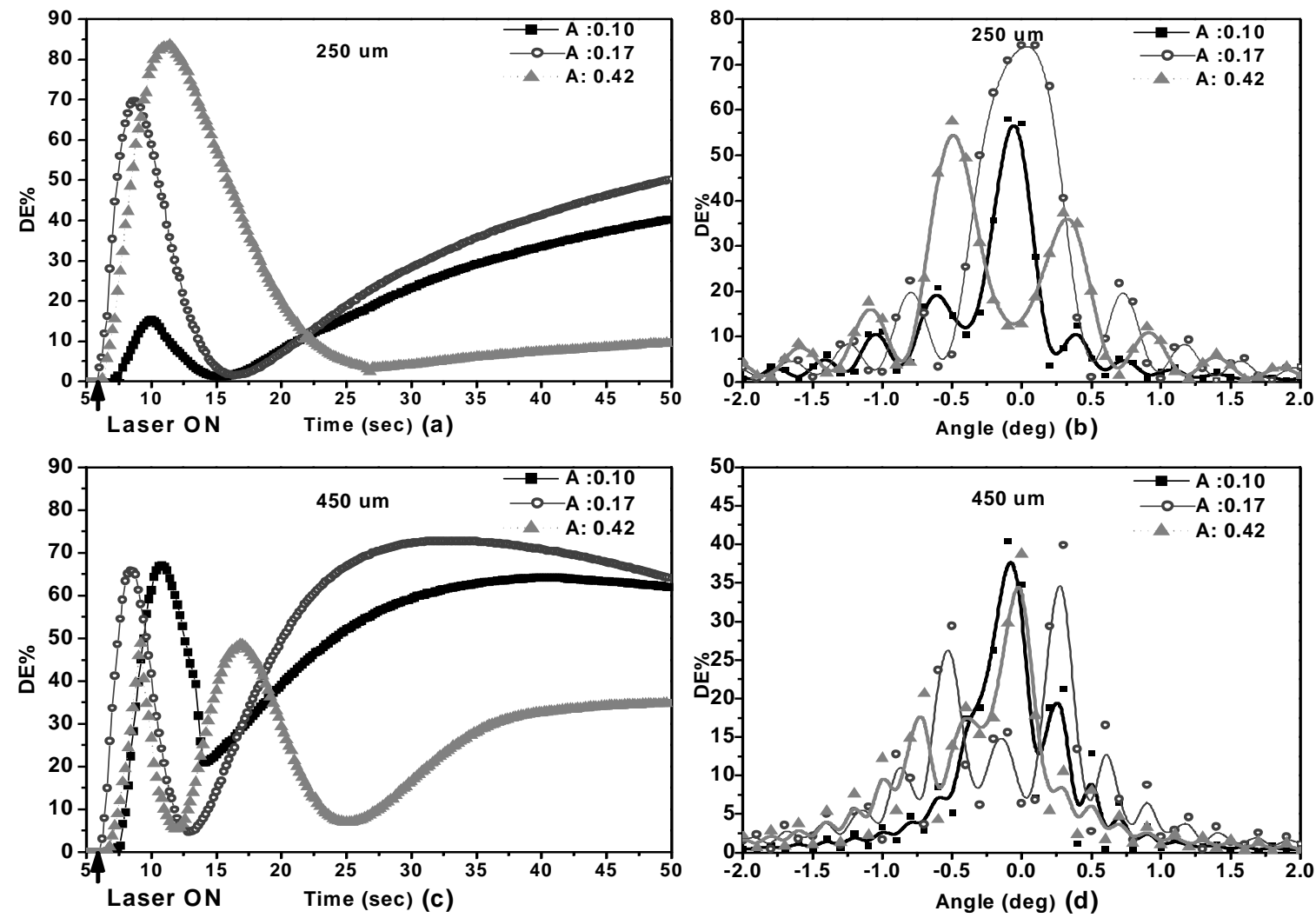

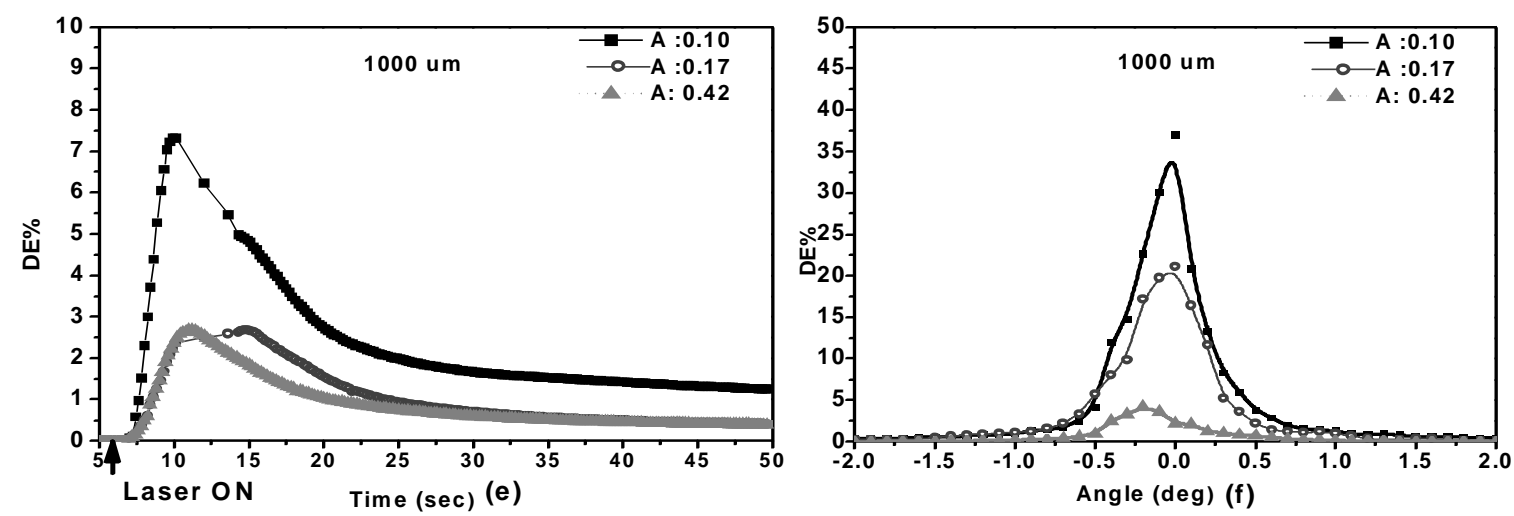

Fig 6: DE vs. exposure time (a, c, e) and angular response (b, d, f) for $\mathrm{A}_{532 \mathrm{~nm}}$ of $0.10(\mathbf{\square}, 0.17(\bullet)$ and $0.42(\boldsymbol{\Delta})$ for layer thicknesses of 250,450 and $1000 \mu \mathrm{m}$ at spatial frequency of 1000 lines $/ \mathrm{mm}$ and intensity $5 \mathrm{~mW} / \mathrm{cm}^{2}$.

It is seen that for smaller thickness the maximum value of DE was reached at the highest value of absorbance. At low absorbance the polymerization is probably not effective enough due to low absorption. As the thickness increases the DE starts to decrease with increasing absorbance. The probable reason is that at higher absorbances the noise grating due to scatting effect becomes stronger and its influence is more pronounced. At layer thickness $1000 \mu \mathrm{m}$ the DE significantly drops for all three absorbances due to increasing scattering. In order to analyze further the results presented in Fig. 6 we have plotted the values of the first peak of the first order diffraction efficiency versus the thickness of the layers for the three different values of layers absorbance (see Fig.7).

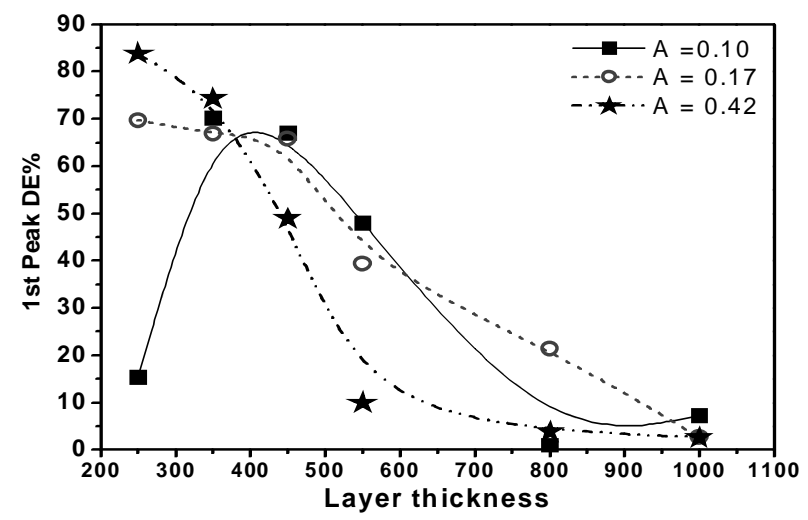

Fig 7: First peak of the first order DE vs. layer thickness for different absorbances.

It is seen that with decreasing layers absorbance the thickness values above which DE drops significantly increases. The critical thickness of the photopolymer layers for each different 
application would depend on the optical loss that could be tolerated. If we define the critical thickness as the layer thickness above which the optical losses exceed 50\% from the results presented in Fig.7 we can determine that the critical thickness for $A_{532 \mathrm{~nm}}=0.10$ is $550 \mu \mathrm{m}$, for $\mathrm{A}_{532 \mathrm{~nm}}=0.17$ it is $500 \mu \mathrm{m}$ and $450 \mu \mathrm{m}$ for $\mathrm{A}_{532 \mathrm{~nm}}=0.42$.

\subsection{Scatter measurement}

Scatter was characterized by using an integrating sphere (Perkin-Elmer Lambda 900) for a wide range of wavelengths $(400-700 \mathrm{~nm})$. Fig.8 shows the scatter percentage at $532 \mathrm{~nm}$ as a function of different layers thicknesses at three values of layer absorbance. It is seen that the scattering losses increase both with increasing layer thickness and the dye concentration.

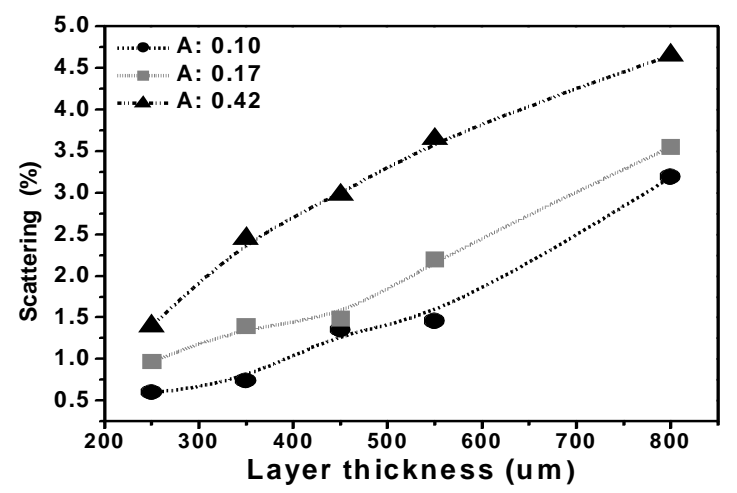

Fig.8: Scattering versus layer at different optical absorptions at 532nm.

Additionally, the scattering of the photopolymer layers was studied using the diffraction patterns. Fig. 9 presents the scattering patterns due to a single probe beam as a function of thickness and absorbance of the layers. A screen was placed at a distance of approximately $10 \mathrm{~cm}$ from the samples to obtain these images. The bright spot on the right side of the image corresponds to the transmitted beam whereas the less intense spot on the left side is the first-order diffracted beam. The transmitted beam diffuses to form a ring pattern indicating the presence of a noise grating [28-30, 37]. Fig.9. shows the increasing intensity of the ring structure with thickness and absorbance. Forshaw at al. [36, 37] explained the rings in terms of the Ewald sphere construction. The intersection of the Ewald sphere with the hemisphere of the primary and its conjugate image, (i.e. the region in reciprocal space spanned by the recorded grating vectors), creates two cones [36-38]. The projections of these cones then produce two bright rings on a screen. 


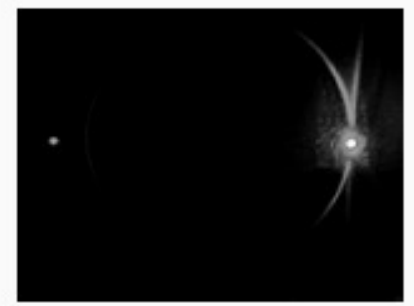

(l)

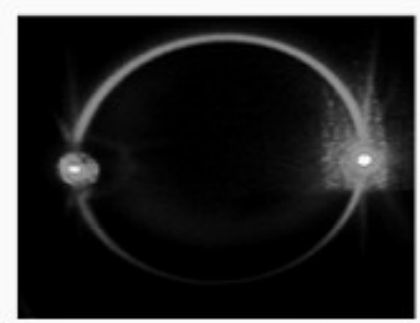

(IV)

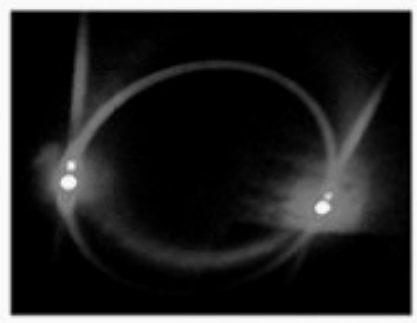

(VII)

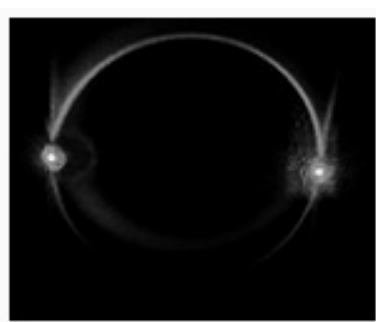

(II)

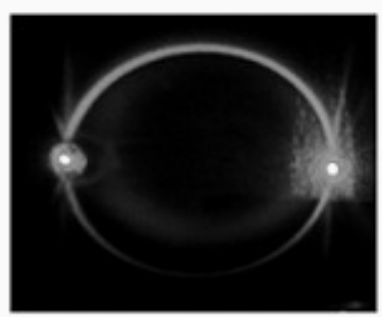

(

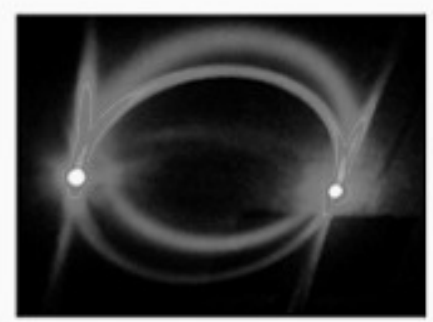

(VIII)

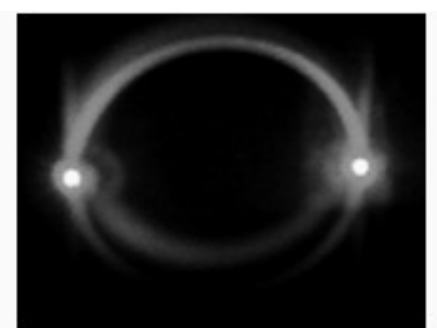

(III)

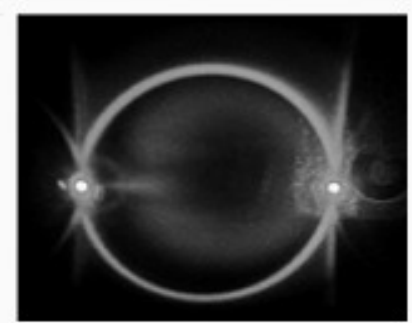

(Vl)

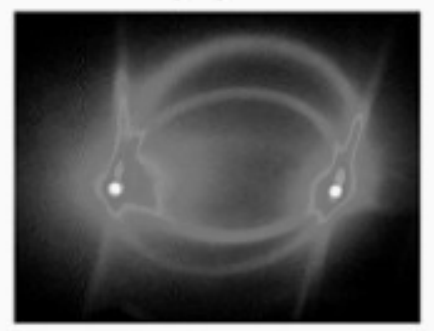

(IX)

Fig.9: Scattering patterns observed in two-beam recording setup for $A_{532 \mathrm{~nm}}$ of 0.10 (top), 0.17 (middle) and 0.42 (bottom row) of layer thicknesses, (I, IV, VII) $250 \mu \mathrm{m}$, (II, V, VIII) $450 \mu \mathrm{m}$, and (III, VI, IX) $800 \mu \mathrm{m}$ : exposure intensity, $5 \mathrm{~mW} / \mathrm{cm}^{2}$, and exposure time $100 \mathrm{~s}$.

The apex angle, $\delta$, of the diffraction cones derived from the Ewald-sphere construction is calculated from the following equation:

$$
\delta=2 \times \arctan \left[\sin \theta /\left(\cos \theta \pm \lambda_{p} / \lambda_{r}\right)\right]
$$

where the recording beam wavelength, $\lambda_{p}$ is $532 \mathrm{~nm}$ and the probe (readout) beam wavelength $\lambda_{\mathrm{r}}$ is $633 \mathrm{~nm}$ and $\theta$ is the readout angle measured with respect to the readout beam within the medium. The positive sign is assigned to the conjugate sphere and the negative sign to the primary sphere. The apex angle depends on the read-out angle, $\theta$, and the probe beam wavelength, $\lambda_{r}$ with respect to the pump beam, $\lambda_{p}$ configuration $\left(\theta=0^{0}\right)$ and reduces to $\delta=\theta$ if $\lambda_{p}=\lambda_{r}$ for the conjugate sphere. The position of the maximum of scattering depends on the ratio $\lambda_{p} / \lambda_{r}$ and the sample thickness, $d$. With increasing thickness the value of $\theta$ approaches $O^{0}$, and therefore scattered intensity increases (Fig.9). Another important feature is that the sign of the 
minimum position of transmission, $\theta$ (for a sufficiently thick sample) depends on whether $\lambda_{p}>$ $\lambda_{r}$ or $\lambda_{p}<\lambda_{r}[38]$.

As shown above the strength of the noise grating in thick photopolymer layers is decreased by lowering the absorbance of the layer. However, our studies on thick samples $(1000 \mu \mathrm{m})$ showed that absorbance at $532 \mathrm{~nm}$ has optimum values around 0.1 . Further decrease of absorbance leads to difficulties in recording because of less photosensitive dye present inside the photopolymer. Scattering from inhomogeneities in layers is the main cause of the parasitic gratings so another possible way to increase $\mathrm{DE}$ for thick layers is to reduce the number of these inhomogeneities by optimizing the photopolymer composition (different PVA for example). These studies are in progress in our group and the results will be published in future.

\section{Conclusion}

Thick acrylamide-based photopolymer layers (up to $1 \mathrm{~mm}$ thick) were prepared and characterized by recording unslanted transmission holographic gratings. The characteristics of acrylamidebased photopolymer layers with thickness from $250 \mu \mathrm{m}$ to 1-mm with the same absorbance were investigated. Real time diffraction efficiency growth curves and angular selectivity profiles were obtained. The dependence of the first order DE on the layer thickness and absorbance was determined. Optimum absorbance at given layer thickness was discussed. By measuring the diffraction efficiency growth of the first diffraction order and studying the diffraction pattern, the influence of scattering on the diffraction efficiency of a thick volume holographic grating was analyzed. The critical thicknesses for acrylamide-based photopolymer layers were determined for different absorbances of the layers. It was found that as the absorbance increases, the critical thickness of the layer decreases. Scattering effects are the main reason for these thickness limitations that should be taken into account when different applications are envisaged.

\section{Acknowledgement}

DIT provided financial support for this project. The authors thank E. Leite, and Q. Cheng (DIT).

\section{References}

1. D. H. Close, A. D. Jacobson, J. D. Margerum, R. G. Brault, and F.J. McClung, 'Hologram recorded on photopolymer holographic recording material,' Appl. Phys. Lett.14, 159-160, 1969. 
2. W. S. Colburn and K. A. Haines, 'Volume Hologram Formation in Photopolymer Materials', Appl. Opt. 10, 1636, 1971.

3. J. M. Moran and I. P. Kaminow, 'Properties of holographic gratings photoinduced in polymethyl methacrylate', Appl. Opt.12, 1964-1970, 1973.

4. W. K. Smothers, B. M. Monroe, A. M. Weber, and D. E. Keys, "Photopolymers for holography," in Practical Holography IV, S. A. Benton, ed., Proc. SPIE 1212, 20-29, 1990.

5. S. Martin, C. A. Feely, and V. Toal, 'Holographic recording characteristics of an acrylamidebased photopolymer,' App. Opt. 36, 5757-5768, 1997.

6. R. Jallapuram, I. Naydenova, S. Martin, R. Howard, V. Toal, S. Frohmann, S. Orlic, H.J. Eichler, 'Acrylamide-based photopolymer for micro holographic data storage', Optical Materials 28, pp: 1329-1333, 2006.

7. D. A. Walkman, H-Y. S. Li and M. G. Horner, 'Volume Shrinkage in Slant Fringe Gratings of a Cationic Ring-Opening Holographic Recording Material,' J. Im. Science and Technology 41, 497-514, 1997.

8. S. Blaya, L. Carretero and A. Fimia, 'Highly sensitive photopolymerisable dry film for use in real time holography', Appl. Phys. Lett.75, 1628-1630, 1998.

9. J. R. Lawrence, F. T. O'Neill and J. T. Sheridan, 'Photopolymer holographic recording material,' Optik, 112, 449-463, 2001.

10. Márquez, C. Neipp, S. Gallego, M. Ortuño, I. Pascual and A. Beléndez, 'Holographically edge enhanced image formation system,' Opt. Lett.28, 1510-1512, 2003.

11. Joel E. Boyd, Timothy J. Trentler, Rajeev K. Wahi, Yadira I. Vega-Cantu, and Vicki L. Colvin, 'Effect of film thickness on the performance of photopolymers as holographic recording materials', Appl. Opt. 39, No. 14, 2353, 2000.

12. C. Zhao, J. Liu, Z. Fu, and R. T. Chen, 'Shrinkage-corrected volume holograms based on photpolymeric phase media for surface-normal optical interconnects,' Appl. Phys. Lett.71, 14641466, 1997.

13. F. T. O'Neill, J. R.Lawrence, J. T. Sheridan, 'Thickness variation of self-processing acrylamide-based photopolymer and reflection holography', Opt. Eng. 40(4), 533-539, 2001.

14. H. J. Coufal, D. Psaltis, G. T. Sincerbox, eds, Holographic Data Storage (Springer-Verlag, New York, 2000.

15. A. Pu and D.Psaltis, 'High-density recording in photopolymer based holographic threedimensional disks,' Appl. Opt. 35, 2389-2398, 1996. 
16. U.-S. Rhee, H. J. Caulfield, J. Shamir, C. S. Vikram, and M. M. Mirsalehi, 'Characteristics of the DuPont photopolymer for angularly multiplexed page-oriented holographic memories,' Opt. Eng. 32, 1839-1847, 1993.

17. J. Ashley, M.-P. Bernal, G. W. Burr, H. Coufal, H. Guenther, J. A. Hoffnagle, C. M. Jefferson, B. Marcus, R. M. Macfarlane, R. M. Shelby, and G. T. Sincerbox 'Holographic Data Storage', IBM Journal of Research and Development, Vol. 44, No. 3, May 2000.

18. H.-Y. S. Li and D. Psaltis, 'Three-dimensional holographic disks,' Appl. Opt. 33, 3764, 1994.

19. M. Ortuño, S. Gallego, C. García, C. Neipp, A. Beléndez and I. Pascual, 'Optimization of a 1 mm thick PVA/acrylamide recording material to obtain holographic memories: method of preparation and holographic properties,' Appl. Phys. B 76, 851-857, 2003.

20. M. Ortuño, S. Gallego, C. García, C. Neipp and I. Pascual, 'Holographic characteristics of a $1 \mathrm{~mm}$ thick photopolymer to be used in holographic memories,' Appl. Opt. 42, 7008-7012, 2003. 21. S. Gallego, M. Ortuño, C. Neipp, A. Márquez, A. Beléndez, I. Pascual, J. V. Kelly and J. T. Sheridan, 'Physical and effective optical thickness of holographic diffraction gratings recorded in photopolymers,' Opt. Express 13 1939-1950, 2005.

22. G. Zhao and P. Mouroulis, 'Diffusion model of hologram formation in dry photopolymer. Materials', J. Mod. Opt. 41, 1929-1934, 1994.

23. S. Piazzolla and B. Jenkins, 'First-harmonic diffusion model for holographic grating formation in photopolymers', J. Opt. Soc. Am. B, Vol-17, 1147-57, 2000.

24. I. Naydenova, R. Jallapuram, S. Martin, R. Howard, V. Toal 'Investigation of Diffusion Processes in a Self-Processing Acrylamide Photopolymer System’ Appl.Opt.43, 2900, 2004.

25. S. Gallego, A. Márquez, D. Méndez, M. Ortuño, C. Neipp, E. Fernández, I. Pascual, and A. Beléndez, 'Analysis of PVA/AA based photopolymers at the zero spatial frequency limit using interferometric methods,' App.Opt. 47, 2557-2563, 2008.

26. V.Moreau, Y.Renotte, Y.Lion, 'Characterization of Du-Pont photopolymer: determination of kinetic parameters in a diffusion model,' Appl. Opt. 41, 3427-3435 (2002).

27. V. L. Colvin, R. G. Larson, A. L. Harris, and M. L. Schilling, 'Quantitative model of volume hologram formation in photopolymers,' J. Appl. Phys. 81, 5913-5923, 1997.

28. L.Carretero, S. Blaya, R. Mallavia, R. F. Madrigal, A. Fimia, 'A theoretical model for noise gratings recorded in acrylamide photopolymer materials used in real-time holography', J. of Mod. Opt., Vol: 45, No.11, 2345-2354, 1998. 
29. X. A. Beléndez, R. Fuentes, and A. Fimia, 'Noise gratings in thick-phase holographic lenses,' J. Opt. Paris 24, 99-105, 1993.

30. Naoaki Suzuki and Yasuo Tomita, 'Holographic scattering in $\mathrm{SiO} 2$ nanoparticles-dispersed photopolymer films', 6809 APP. Opt, Vol. 46, No. 27, pp:6809-6814, 2007.

31. Naoaki Suzuki and Yasuo Tomita, 'Holographic scattering in $\mathrm{SiO} 2$ nanoparticles-dispersed photopolymer films', 6809 APP. Opt, Vol. 46, No. 27, pp:6809-6814, 2007.

32. M. A. Ellabban, M. Fally, M. Imlau, T. Woike, R. A. Rupp, and T. Granzow, 'Angular and wavelength selectivity of parasitic holograms in cerium doped strontium barium niobate', J. Appl. Phys. 96, 6987, 2004.

33. Ellabban M A, Fally M, Ur`sic $\mathrm{H}$ and Dreven`sek-Olenik.I, 'Holographic scattering in photopolymer-dispersed liquid crystals', Appl. Phys. Lett. 87, 151101, 2005.

34. M. A. Ellabban, R. A. Rupp, and M. Fally, 'Reconstruction of parasitic holograms to characterize photorefractive Materials', Appl. Phys. B: Lasers Opt. 72,635, 2001.

35. H. Kogelnik 'Coupled Wave Theory for Thick Hologram Gratings', Bell Syst. Tech. J. 48, 2909, 1969.

36. M. R. B. Forshaw, 'Explanation of the Venetial blind effect in holography using the Ewald sphere concept', Opt. Communication. 8, 201-206, 1973.

37. M. R. B. Forshaw, 'Explanation of the two-ring diffraction phenomenon observed by Moran and Kaminow,' Appl. Opt. 13, 2, 1974.

38. R. Magnusson and T. K. Gaylord, 'Laser scattering induced holograms in lithium niobate,' Appl. Opt. 13, 1545-1548 (1974). 